\title{
Ureteroscopy-Assisted Retrograde Nephrostomy for Percutaneous Nephrolithotomy after Urinary Diversion
}

\author{
Takashi Kawahara $^{a, b}$ Hiroki Ito $^{a, b}$ Hideyuki Terao $^{a}$ \\ Takehiko Ogawa $^{\text {b Hiroji Uemura }}{ }^{\text {boshinobu Kubota }}{ }^{b}$ \\ Junichi Matsuzaki ${ }^{\mathrm{a}}$ \\ ${ }^{a}$ Department of Urology, Ohguchi Higashi General Hospital, and ${ }^{\mathrm{b}}$ Department of Urology, \\ Yokohama City University Graduate School of Medicine, Yokohama City, Japan
}

\section{Key Words}

Ureteroscopy - lleal conduit - Lawson catheter - Retrograde nephrostomy ·

Urinary diversion

\begin{abstract}
A 33-year-old male with an ileal conduit was referred to our department for the treatment of left renal calculi. After inserting a ureteral access sheath, a ureteroscopy-assisted retrograde nephrostomy was made. Percutaneous nephrolithotomy was successfully achieved using this nephrostomy.
\end{abstract}

\section{Introduction}

The implementation of a diversion that requires the use of an appliance, such as an ileal conduit or cutaneous ureterostomy, is primarily considered for patients undergoing radical cystectomy who are not candidates for continent diversion procedures [1]. However, a significant number of patients have complications associated with ileal conduit diversion. In addition, at a later stage, ureteral calculi have been reported to develop in $10.7 \%$ of patients, while uretero-ileal obstruction has been observed in 4 to $7.9 \%$ of cases [2].

Goodwin et al. [3] first reported percutaneous renal access in 1955. After that, percutaneous nephrolithotomy (PCNL) was developed and has become the standard procedure for large renal stones. Ultrasound-guided puncture of the renal collecting system with subsequent placement of a drainage tube under fluoroscopic guidance is 
the standard modality for percutaneous nephrostomy. However, ultrasonographic- or fluoroscopic-guided puncture is difficult for the cases with a non-dilated renal collecting system.

Even when percutaneous access is successfully gained with a needle puncture in the non-dilated collecting system, the tract is often not in the most desirable location for stone extraction [4]. Retrograde nephrostomy was first developed by Lawson et al. [4] in 1983, and Hunter et al. [5] reported 30 cases of retrograde nephrostomy in 1985. With this procedure, after the needle has exited through the skin, no further steps are required to prepare for the dilation.

We previously described ureteroscopy (URS)-assisted retrograde nephrostomy (UARN) [6]. In UARN it is possible to continuously visualize the dilation from puncture to insertion of the nephroaccess sheath (NAS). We herein report the first case of UARN for a patient who underwent ureteral diversion.

\section{Case Presentation}

A 33-year-old male with an ileal conduit due to spinal bifida was referred to our department for the treatment of a $1.7-\mathrm{cm}$ left renal calculus. CT did not detect the renal stone or indicate whether it was in the renal collecting system or the renal parenchyma. However, using URS before making the nephrostomy, it was possible to confirm that the renal stone was in the renal collecting system.

Under general anesthesia, the patient was placed in a Galdakao-modified Valdivia position. A flexible cystoscope (Olympus, Tokyo, Japan) was inserted into the ileal conduit and approached the left ureteral orifice. After inserting a guidewire into the left ureter, retrograde uretero-pyelography was performed as shown in fig. 1a. Next, an 11/13 Fr (inner/outer diameter) ureteral access sheath (Navigator ${ }^{\circledR} 11$ Fr $46 \mathrm{~cm}$; Boston Scientific, Natick, Mass., USA) was inserted into the ureter under fluoroscopic guidance. We carefully observed the target calculus using a flexible URS (Flex-X2 ${ }^{\circledR}$; Karl Storz, Tuttlingen, Germany) and defined the appropriate position to puncture with assistance provided by pyelography. Thereafter, a Lawson retrograde nephrostomy puncture wire (COOK Urological, Bloomington, Ind., USA) without a coating cover was set into the flexible URS. The URS was approached to the desired calix again, and the puncture wire was moved toward the route from the target calix to the exit skin under fluoroscopic assistance (fig. $1 \mathrm{~b}$ and fig. 2 a).

To avoid injuries to the spleen, intestines, and pleural cavity, the puncture was performed after ultrasonography. The puncture wire was passed through the muscle easily and 'tented' the skin at the posterior axially line. The skin was then incised, and the needle was delivered. Next, the dilator was placed using the puncture wire, which was advanced through the skin, subcutaneous fat, abdominal wall musculatue, and perinephic fat until it reached the renal parenchyma under visualization with flexible URS. A one-step 18.5 Fr dilator (Karl Storz) was inserted under visualization with flexible URS and fluoroscopy after serial dilation (fig. $2 \mathrm{~b}$ ). We simultaneously viewed the parenchyma with URS and nephroscope for $22 \mathrm{~min}$. Stone fragments were removed through the percutaneous NAS, and the stone fragments which the nephroscope could not reach were repositioned by flexible URS. Each urologist on each side controlled the scope to avoid damage to the opposite scope.

The nephrostomy was removed at the end of the operation, and a ureteral stent (Polaris Ultra ${ }^{\circledR}$ $8 \mathrm{Fr} 30 \mathrm{~cm}$; Boston Scientific) was placed at the conclusion of the operation. Tubeless PCNL was achieved, and no intra- or preoperative complications were observed. The ureteral stent was removed 2 weeks after the operation, and the patient was confirmed to be stone-free by postoperative kidneyureter-bladder films. A chemical analysis revealed that the stone was composed of calcium oxalate monohydrate. 


\section{Discussion}

Retrograde nephrostomy was first developed by Lawson et al. [4] in 1983, and Hunter et al. [5] reported 30 cases of retrograde nephrostomy in 1985. Using this procedure, after the needle exits through the skin, no further steps are required in preparation for dilation. In the original Lawson retrograde nephrostomy procedure, positioning and puncturing are only possible under fluoroscopic guidance, so adjusting the ideal angle and puncturing on demand was not possible. As a result, the Lawson technique failed to gain widespread acceptance.

We described the UARN technique as a new strategy for PCNL in 2011 [6]. UARN is performed in a Galdakao-modified Valdivia position [7]. In 1987, Valdivia-Uria described a PCNL with the patient in the supine position, with a 3-litre serum bag placed below the flank [7]. Using this position, both surgical and anesthesiological advantages have been described. In 2001, Ibarluzea et al. [7] reported a Galdakaomodified Valdivia position in which the supine position is the same as that used in the Valdivia position, but the leg of the target side is extended, while the contralateral leg is abducted. This position has the advantage of allowing simultaneous percutaneous and retrograde access [7]. An occlusion catheter under ultrasonographic and fluoroscopic guidance is widely used for dilating the renal collecting system in order to avoid puncturing the tubules. We continuously visualized the motion of the ureteroscope under ultrasonography and were able to easily detect the tent sign without changing the patient's position.

A significant number of patients have complications associated with ileal conduit diversion. As described by Schmidt et al. [8], in the later postoperative periods, ureteral calculi develop in $10.7 \%$ of patients. Turk et al. [9] reported that urinary stone formation is one of the more common adverse events and can develop in the upper urinary tract or within the diversion itself in up to $11 \%$ of patients within 3 years after surgery.

An antegrade or retrograde approach can be used to achieve access to the ureter in individuals with ileal conduit urinary diversion [10]. URS is an attractive procedure; however, because of the complex anatomical structure after urinary diversions, which often makes it difficult to reach the target calyx, PCNL is often considered as the treatment of first choice to achieve a stone-free status in patients with urinary diversions and large or complex upper tract calculi. However, in non-dilated renal collecting systems, puncturing and inserting the guidewire into the ureter is sometimes challenging. We treated another 3 patients with ileal conduit in the Bricker fashion. In these patients, the ureteral access sheath was also successfully inserted without any difficulty.

With this procedure, after the needle has exited through the skin, no further steps are required in preparation for dilation. In addition, UARN facilitates continuous visualization from puncture to insertion of the NAS by flexible URS. 


\section{Conclusions}

UARN was successfully used for a patient who underwent ileal conduit urinary diversion. UARN might provide a new option for patients with a non-dilated renal collecting system after urinary diversion.

\section{Disclosure Statement}

None of the authors have a conflict of interest to declare.

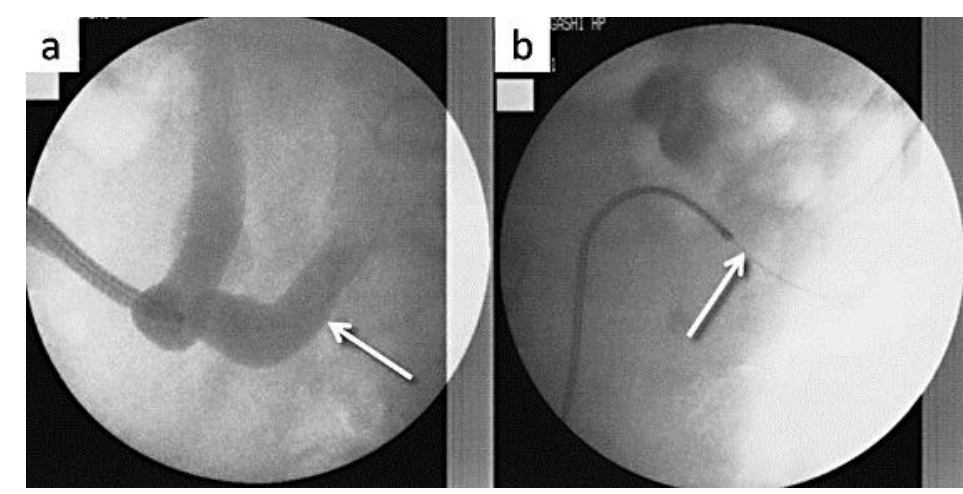

Fig. 1. Retrograde pyelography from the ileal conduit (a) and puncturing from the renal collecting system to the skin (b).

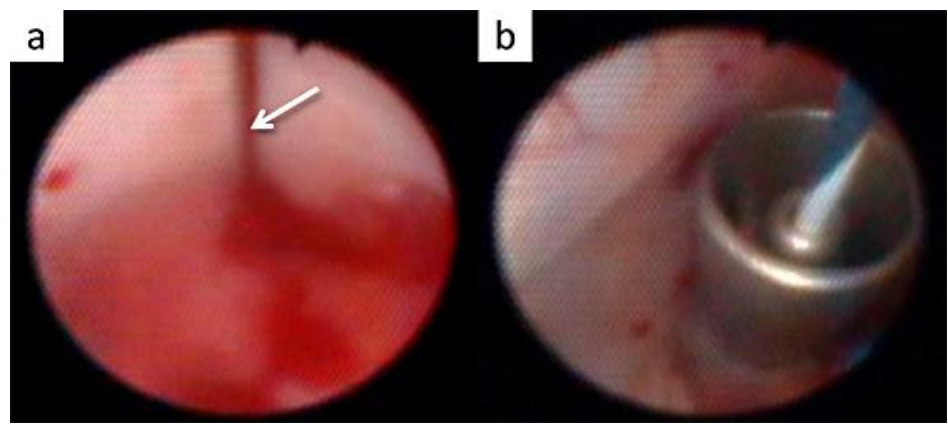

Fig. 2. The puncture wire was advanced from the URS (a), and the NAS was inserted using a one-step dilator (b). 


\section{References}

1 Rodriguez AR, Lockhart A, King J, Wiegand L, Carrion R, Ordorica R, Lockhart J: Cutaneous ureterostomy technique for adults and effects of ureteral stenting: an alternative to the ileal conduit. J Urol 2011;186:1939-1943.

2 Vandenbroucke F, Van Poppel H, Vandeursen H, Oyen R, Baert L: Surgical versus endoscopic treatment of non-malignant uretero-ileal anastomotic strictures. Br J Urol 1993;71:408-412.

-3 Goodwin WE, Casey WC, Woolf W: Percutaneous trocar (needle) nephrostomy in hydronephrosis. J Am Med Assoc 1955;157:891-894.

4 Lawson RK, Murphy JB, Taylor AJ, Jacobs SC: Retrograde method for percutaneous access to kidney. Urology 1983;22:580-582.

5 Hunter PT, Finlayson B, Drylie DM, Leal J, Hawkins IF: Retrograde nephrostomy and percutaneous calculus removal in 30 patients. J Urol 1985;133:369-374.

6 Kawahara T, Ito H, Terao H, Yoshida M, Ogawa T, Uemura H, Kubota Y, Matsuzaki J: Ureteroscopy assisted retrograde nephrostomy: a new technique for percutaneous nephrolithotomy (PCNL). BJU Int 2012;110:588-590.

-7 Ibarluzea Gonzalez G, Gamarra Quintanilla M, Gallego Sanchez JA, Pereira Arias JG, Camargo Ibargaray I, Bernuy Malfaz C: Percutaneous kidney lithotripsy. Clinical course, indications, and current methodology in our Lithotripsy Unit (in Spanish). Arch Esp Urol 2001;54:951-969.

-8 Schmidt JD, Hawtrey CE, Flocks RH, Culp DA: Complications, results and problems of ileal conduit diversions. J Urol 1973;109:210-216.

-9 Turk TM, Koleski FC, Albala DM: Incidence of urolithiasis in cystectomy patients after intestinal conduit or continent urinary diversion. World J Urol 1999;17:305-307.

10 Drake MJ, Cowan NC: Fluoroscopy guided retrograde ureteral stent insertion in patients with a ureteroileal urinary conduit: method and results. J Urol 2002;167:2049-2051. 